\section{MS08 O4}

Preparing a Laue experiment Annemarie Wöhri ${ }^{\mathrm{a}}$, Magnus Andersson ${ }^{\mathrm{a}}$, Erik Malmerberg ${ }^{\mathrm{b}}$, Pia Wadsten ${ }^{\mathrm{a}}$ and Richard Neutze ${ }^{\mathrm{b} a}$ Department of Chemical and Biological Engineering, Chalmers University of Technology, 40530 Gothenburg, Sweden. ${ }^{b}$ Department of Chemistry Biochemistry and Biophysics, Gothenburg University, 40530 Gothenburg, Sweden.

E-mail: wohri@,chembio.chalmers.se

\section{Keywords: laue diffraction, sponge phase crystallization, membrane proteins}

Photosynthesis is the process by which light is converted into chemical energy. In Rhodobacter sphaeroides the light-driven reaction takes place in the membrane-bound protein complex called the reaction centre (RC). The photocycle starts with capture of a photon by the special pair $(\mathrm{P})$ followed by a transmembrane electron transfer reaction. The final electron acceptor is a mobile ubiquinone molecule $\left(\mathrm{Q}_{\mathrm{B}}\right)$ and a $\mathrm{P}^{+} \mathrm{Q}_{\mathrm{B}}{ }^{-}$charge separated state is formed. After absorption of a second photon the ubiquinone becomes fully reduced and diffuses through the membrane where it gets oxidised. As a consequence a proton gradient across the membrane will occur and the energy stored in this gradient is used for synthesis of ATP. Even if RC is the first membrane protein with known structure its photocycle is still not fully understood and structural details are unknown. Controversy exists whether or not the formation of the $\mathrm{P}^{+} \mathrm{Q}_{\mathrm{B}}{ }^{-}$state is coupled to light induced conformational changes. To get more structural insight one can perform a time-resolved laue diffraction experiment, where structural changes can be monitored as a function of time. To be able to achieve such an experiment a few requirements have to be met. A prerequisite is a well studied system, whose reaction cycle can be induced by a laser source and which is able to relax back rather quickly, which is the case in RC. Furthermore for the studies of $\mathrm{RC}$ one needs to investigate the illumination conditions necessary to induce the $\mathrm{P}^{+} \mathrm{Q}_{\mathrm{B}}{ }^{-}$state within the crystal without destroying the crystal, the life time of the induced state and the average occupancy of $\mathrm{Q}_{\mathrm{B}}$ in the active site. Knowledge about these parameters has already been obtained by performing a pump-probe measurement on single RC crystals using a microspectrophotometer and a nanosecond laser. On top of all this requirements the main demand is still well diffracting, robust crystals, where the protein is in its active form. In previous work crystals of RC diffracting to $2.2 \AA$ have been obtained using a new crystallization method called the lipidic sponge phase crystallisation [1]. The crystals grown in this liquid phase were arranged as stacked 2D bilayers, are robust, active, of high quality and superior to detergent grown crystals and therefore ideal for timeresolved laue experiments. Since a lot of crystals are needed and the experiment is carried out at room temperature fishing and mounting is not trivial. Therefore a fishing protocol has been worked out protecting the crystals from dehydration. So fare we have fulfilled our requirements and first laue diffraction experiments have been performed. From this data a ground state dataset diffracting to $2.6 \AA$ has been obtained and hopefully light induced data will follow soon.
[1] Wadsten, P., Wöhri, A.B., Snijder, A., Katona, G., Gardiner, A.T., Cogdell, R.J., Neutze, R. \& Engström, S. (2006). Lipidic sponge phase crystallization of membrane proteins. J. Mol. Biol. $364,44-53$.

\section{MS08 O5}

Ultrafast structural dynamics: a simulated timeresolved $\mathrm{X}$-ray scattering experiment of liquid halorhodopsin Magnus Andersson ${ }^{a}$, Jonathan Vincent ${ }^{b}$, David van der Spoel $^{c}$, Richard Neutze ${ }^{d}{ }^{a}$ Department of Chemical and Biological Engineering, Chalmers University of Technology. ${ }^{b}$ Department of Photochemistry and Molecular Science, Uppsala University. ${ }^{c}$ Department of Cell and Molecular Biology, Uppsala University. ${ }^{d}$ Department of Chemistry Göteborg University. Email: magnus.andersson@chalmers.se

Keywords: time-resolved scattering studies, molecular dynamics simulations, membrane proteins

Liquid phase time-resolved X-ray scattering as a tool for directly observing structural changes of photoactive chemicals in solution has attracted extensive attention $\left(\mathrm{CH}_{2} \mathrm{I}_{2}\right.$ [1], $\mathrm{CH}_{2} \mathrm{Cl}_{2}$ [2]). It is important to extend this methodology to incorporate light-driven proteins in order to understand light-induced changes structurally since they might reveal the underlying mechanism by which the entire protein functions. Pump and probe experiments are based on the offset of a reaction by a laser pulse, which is allowed to propagate for certain time intervals probed by isolated X-ray pulses. Given that the laser pulse only excites a small fraction of the photo-chemicals and the influence of the solvent on the signal, it becomes clear that signal-to-noise is an important issue. Therefore, a prerequisite for studying light-induced reactions of a protein in solution is the existence of heavy elements in the system functioning as markers, about which local structural changes will be resolved with time. The membrane protein halorhodopsin is an attractive model system due to its ability to incorporate several heavy atoms and the fact that its photocycle is not as well studied as its close relative, bacteriorhodopsin. This work anticipates the outcome of a time-resolved X-ray scattering experiment on halorhodopsin. Radial distribution functions were extracted from a series of molecular dynamics simulations (GROMACS) and scattering intensities were calculated. This method is only dependent on the interatomic distances of the specific markers, and is therefore not limited to chromophore systems with favourable spectroscopic properties. By extending the methodological principles laid out in this work, time resolved experiments of halorhodopsin and other light-driven membrane proteins, lies within grasp.

[1] Davidsson J., Poulsen J., Cammarata M., Georgiou P., Wouts R., Katona G., Jacobson F., Plech A., Wulff M., Nyman G., and Neutze R. Structural determination of a transient isomer of $\mathrm{CH}_{2} \mathrm{I}_{2}$ by picosecond X-ray diffraction. Physical Review Letters 94 245503 (2005).

[2] Georgiou P., Vincent J., Andersson M., Wöhri A.B., Gourdon P., Poulsen J., Davidsson J. and Neutze R. Picosecond calorimetry: Time-resolved $\mathrm{x}$-ray diffraction studies of liquid $\mathrm{CH}_{2} \mathrm{Cl}_{2}$. The Journal of Chemical Physics 124, 1 (2006). 